\title{
Barriers to Post-Acute Care for Patients on Opioid Agonist Therapy; An Example of Systematic Stigmatization of Addiction
}

\author{
Sarah E. Wakeman, $M D^{1,2,3}$ and Josiah D. Rich, MD, MPH $H^{3,4,5}$ \\ 'Massachusetts General Hospital, Boston, MA, USA; ${ }^{1}$ Harvard Medical School, Boston, MA, USA; ${ }^{3}$ Center for Prisoner Health and Human Rights, \\ Providence, RI, USA; ${ }^{4}$ Warren Alpert Medical School of Brown University, Providence, RI, USA; ${ }^{5}$ Division of Infectious Diseases, the Miriam Hospital, \\ Providence, RI, USA.
}

J Gen Intern Med 32(1):17-9

DOI: $10.1007 / \mathrm{s} 11606-016-3799-7$

(c) Society of General Internal Medicine 2016

$\mathrm{O}$ pioid use disorder is a public health crisis. In 2014, 1.9 million Americans had a prescription opioid use disorder and 586,000 had a heroin use disorder. ${ }^{1}$ Despite the prevalence of opioid use disorder, stigma continues to be a major barrier to effective treatment. Addiction is the most stigmatized condition on earth. Stigma prevents individuals from seeking treatment and is associated with poor mental and physical health. There is additional stigma related to different types of treatment. Maintenance therapy with opioid agonists, methadone or buprenorphine, is the most effective treatment for opioid use disorder, reducing death and relapse by 50 percent. Despite this evidence, stigma and misunderstanding about medication treatments persist. Practical examples of this abound. Only a minority of physicians are trained to prescribe buprenorphine, most addiction programs do not offer pharmacotherapy, and patients treated with medications are often not accepted to addiction treatment programs or to post-acute medical facilities. This last barrier is of particular relevance to patients who inject opioids and are at higher risk of medical conditions requiring post-acute care.

Among individuals with opioid use disorder, those with a history of injection drug use (IDU) face particular health risks, especially infectious complications. A study of hospitalized patients with a history of IDU found that $47 \%$ had at least one infectious complication. ${ }^{2}$ Many infectious diseases associated with IDU, such as endocarditis or osteomyelitis, require weeks of intravenous antibiotic therapy. The incidence of endocarditis-related hospitalizations in particular has grown over the past decade, and a majority of endocarditis cases in urban settings are related to IDU. Patients without a history of drug use may receive treatment at home or in a skilled nursing facility (SNF). For those with a history of IDU, post-acute care

Received September 2, 2015

Revised December 4, 2015

Accepted June 23, 2016

Published online July 8, 2016 treatment options are often limited. Healthcare facilities and infusion companies may not allow people with a history of IDU to have home intravenous antibiotic therapy because of concern that an indwelling intravenous catheter could be used to inject illicit drugs. Many SNFs will not accept a patient with a history of IDU even if the individual has begun treatment with opioid agonists. Even those on opioid agonist therapy and no history of IDU are routinely rejected from SNFs. These barriers have led to pilot interventions exploring the administration of antibiotics within a residential addiction treatment program. ${ }^{3}$

While such alternative models to provide both addiction treatment and post-acute medical care are promising, few exist. Consequently, most patients with opioid use disorder and infectious diseases are left with limited options for SNF placement. In addition, hospitals bear substantial costs, as patients often remain in the acute care setting for longer than expected, sometimes until the entire course of antibiotics is completed. ${ }^{3}$ Not surprisingly, a model of delivering antibiotics within a residential addiction treatment program was shown to save 2.43 million dollars for the care of 205 patients. $^{3}$

Historically, patients with other stigmatized illnesses have similarly been excluded from post-acute care settings. HIV/ AIDS is an unfortunate recent example of this type of exclusion. A 2003 study found that $46 \%$ of SNFs surveyed in LA County did not accept patients with HIV/AIDS. ${ }^{4}$ A majority gave no reason for this policy. An example of one respondent's reply to why the facility did not accept patients with HIV/ AIDS was "We try not to...I'm just trying to be honest."4 Among those facilities that did offer an explanation, the most common reasons included not having taken a patient with HIV/AIDS before, inadequate staff training, no procedures in place to care for such patients, or that they had a policy of only taking elderly patients. ${ }^{4}$ These practices were found to be in violation of federal disability discrimination laws.

There are no surveys to document how prevalent the exclusion of patients with opioid use disorder from SNFs is. However, this appears to be the norm for patients on opioid agonist therapy, at least in the northeast where we practice. At a 2015 meeting of a collaboration of Massachusetts SNFs, none reported accepting such patients at that time. Protection from discrimination for individuals with addiction under the Americans with Disabilities Act (ADA) is not as clear as for other diseases. The 
ADA does not consider someone actively using illicit drugs as a "qualified individual with a disability," but those in recovery and those currently participating in treatment and no longer using drugs are protected under the ADA. ${ }^{5}$ Individuals on opioid agonist therapy are generally considered to have a disability and to be covered by anti-discrimination laws, including the Americans with Disabilities Act. ${ }^{5}$ Under these protections, the practice of not accepting patients on opioid agonist therapy to a SNF could be considered discrimination.

While blanket exclusion of patients on opioid agonist therapy appears to be a clear violation of anti-discrimination laws, there are logistical and regulatory barriers that create uncertainty and may lead to a facility's rejection. The rules governing methadone and buprenorphine in a SNF are the same as those for a long-term care facility (LTCF). These regulations allow for patients on opioid agonist therapy to continue receiving treatment at a SNF under the following conditions. Patients on methadone maintenance through an opioid treatment program can continue to receive methadone through daily visits to the program. Alternatively, the program can transfer medication to the facility with the approval of the State Methadone Authority. Patients on buprenorphine with a community prescriber can continue with the medication prescribed by their prescriber as for any other home medication not on formulary. Alternatively, a physician at the facility could get waivered to prescribe buprenorphine and prescribe it for any admitted patient with an opioid use disorder. A physician can become waivered to prescribe buprenorphine by completing an 8-h training and registering with the DEA. Lastly, for patients who are appropriate for opioid agonist therapy but do not meet the above criteria, the facility may administer methadone or buprenorphine for up to 3 days to relieve withdrawal symptoms while arrangements are made for referral to treatment. Misunderstanding of these complex regulations may cause facilities to inaccurately state that they are not allowed to care for these patients.

Further steps are needed to comprehensively address the discrimination and deficiencies in post-acute care for individuals with opioid use disorder on agonist therapy. Education about the existing policies will help to a modest degree. However, the regulations are unnecessarily complicated and burdensome, requiring coordination with outside treatment agencies or buprenorphine training that may be unrealistic. Changing federal regulations so that post-acute facilities can provide opioid agonist therapy for patients admitted for a condition other than opioid use disorder, which is the exemption afforded hospitals, would help expand access, minimize disruptions, and decrease barriers to care.

Stronger enforcement of ADA laws as they apply to individuals with opioid use disorder also is an important step that has yet to be taken. This may require legal action on behalf of discriminated patients to realistically create change. Increasing the buprenorphine patient limit (the number of patients for whom a provider can prescribe buprenorphine) and allowing nurse practitioners to prescribe, as proposed recently by President Obama, and supporting the "Recovery Enhancement for Addiction Treatment Act," could help. These changes would permit SNF providers, who may cover several facilities, to care for more patients on buprenorphine and thus make the completion of buprenorphine training more worthwhile. It would also expand access to buprenorphine in the community and allow community prescribers to continue to prescribe without impacting access for new patients.

Physicians can play an important role in advocacy around this issue. Improved understanding of the regulations governing opioid agonist prescribing could help physicians encourage SNFs to admit patients already on buprenorphine, noting that such patients could be continued on therapy by their existing waivered physician. Recognizing when SNF rejections are in violation of the ADA would allow physicians and hospitals to advocate for patients and encourage legal action when necessary. An even more effective policy change would be to remove the need for a waiver entirely; however, this would require changes to existing federal law, the Drug Abuse Treatment Act 2000. In the absence of legislative reform, physicians and health systems can improve access to buprenorphine through the existing process. Buprenorphine saves lives, improves clinical outcomes, and reduces healthcare costs, all of which make an argument to encourage or even mandate physicians to get waivered given the current opioid epidemic. This would allow more patients to initiate buprenorphine in the community and continue on therapy during acute and post-acute care. Additionally, there is strong evidence that initiating opioid agonist therapy during hospitalization improves treatment retention and patient outcomes. Making this the standard of care, so all patients with opioid use disorder are started on agonist therapy in the hospital, coupled with the above policy changes, could create needed systems change and redefine the expectation for how patients with this disease are treated.

Acknowledgments: Dr. Rich is supported by Award No. K24DA022112 from the National Institute On Drug Abuse. The content of this piece is solely the responsibility of the authors and does not necessarily represent the official views of the National Institute On Drug Abuse or the National Institutes of Health. Dr. Rich's research has also been facilitated in part by the infrastructure and resources provided by the Lifespan/Tufts/Brown Center for AIDS Research, an NIH-funded program, grant no. P3O-AI-42853, from the National Institutes of Health, Center for AIDS Research (NIH/CFAR).

Corresponding Author: Sarah E. Wakeman, MD; Massachusetts General Hospital, 55 Fruit Street, Founders 880, Boston, MA 02114 , USA (e-mail: swakeman@partners.org).

\section{Compliance with ethical standards:}

Conflicts of interest: The authors declare that they do not have a conflict of interest. 


\section{REFERENCES}

1. Substance Abuse and Mental Health Services Administration. Behavioral health trends in the United States: results from the 2014 national survey on drug use and health: SAMHSA, 2015.

2. Scheidegger $\mathbf{C}$, Zimmerli W. Infectious complications in drug addicts: seven-year review of 269 hospitalized narcotics abusers in Switzerland.

3. Jewell C, Weaver M, Sgroi C, Anderson K, Sayeed Z. Residential addiction treatment for injection drug users requiring intravenous antibiotics: a costreduction strategy. J Addict Med. 2013;7(4):271-6.
4. Sears B, Ho D. HIV Discrimination in Health Care Services in Los Angeles County: The Results of Three Testing Studies. December 2006. Available online at http://www.bc.edu/content/dam/files/schools/law_sites/library/pdf/content/tremblay_schulman/2006-12-00.Sears_Ho.pdf (Accessed March 14, 2016).

5. United States Commission on Civil Rights. Substance Abuse under the ADA. Available online at http://www.usccr.gov/pubs/ada/ch4.htm (Accessed March 14, 2016). 